\title{
Liga Acadêmica de Nutrição Funcional e Esportiva: uma iniciativa para contribuição na formação dos estudantes de Graduação em Nutrição
}

Academic League of Functional and Sports Nutrition: an initiative to contribute to the training of Undergraduate Nutrition students

Liga Académica de Nutrición Funcional y Deportiva: una iniciativa para contribuir a la formación de estudiantes de Licenciatura en Nutrición

Recebido: 27/01/2021 | Revisado: 02/02/2021 | Aceito: 04/02/2021 | Publicado: 10/02/2021

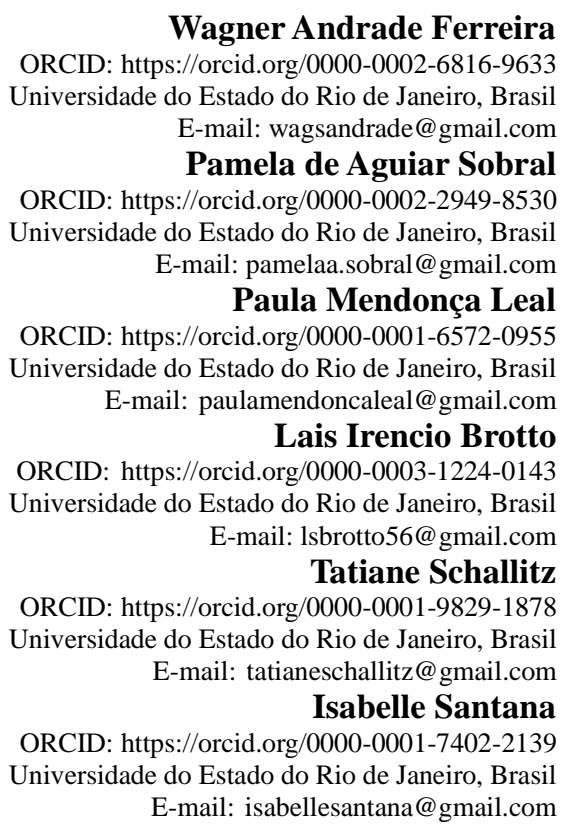

\section{Resumo}

A Liga Acadêmica de Nutrição Funcional e Esportiva (LANFE) foi fundada por acadêmicos com propósito de aprimorar o conhecimento teórico-prático sobre nutrição funcional e esportiva. O objetivo deste estudo foi relatar a experiência de criação e implantação de um projeto de extensão da $1^{a}$ liga acadêmica (LA) em um curso de nutrição de uma universidade pública do Estado do Rio de Janeiro, discorrer sobre as atividades realizadas e avaliar seu impacto. Trata-se de um estudo quali-quantitativo, descritivo, do tipo relato de experiência da criação e primeiro ano de atividades da liga. Na avaliação de questionários aplicados à comunidade interna e externa da universidade, observou-se que 99,2\% dos participantes $(n=123)$ acreditavam na importância da criação de uma LA para o Curso de Nutrição. Quanto às atividades realizadas pela LANFE, 100\% dos participantes $(\mathrm{n}=15)$ do Workshop de gastronomia funcional sobre cacau consideraram o nível de qualidade do evento como "muito bom", bem como o suporte às dúvidas e preocupações expostas. Além disso, 85,3\% dos participantes do I Simpósio da LANFE $(\mathrm{n}=34)$ demonstraram um grau de satisfação "muito bom". Desta forma, a liga acadêmica mostrou-se um importante instrumento de ensino e aprendizagem, mobilizando estudantes e profissionais em prol da construção e do compartilhamento de conhecimentos científicos, contribuindo assim, para a formação dos estudantes de graduação em nutrição.

Palavras-chave: Ligas acadêmicas; Nutrição funcional; Nutrição esportiva; Ensino.

\begin{abstract}
The Academic League of Functional and Sports Nutrition (LANFE) was founded by students in order to improve theoretical and practical knowledge on functional and sports nutrition. The aim of this study was to report the experience of creating and implementing an extension program of the 1st academic league (AL) in a nutrition undergraduation course at a public university in the State of Rio de Janeiro, discuss the performed activities and evaluate their impact. This study is qualitative-quantitative and descriptive type, and reports the creation experience
\end{abstract}


and activities of the league's first year. In the evaluation questionnaires applied to internal and external community, 99.2\% of participants $(n=123)$ believed in the importance of creating an AL for the Nutrition Course. Regarding the activities carried out by LANFE, $100 \%$ of the participants $(n=15)$ of the Workshop on functional gastronomy about cocoa considered the event's quality level to be "very good", as well as the support to the questions and concerns exposed. Furthermore, $85.3 \%$ of LANFE's I Symposium participants $(n=34)$ demonstrated a degree of satisfaction "very good". In this way, the academic league proved to be an important teaching and learning tool, mobilizing students and professionals in favor of the construction and sharing of scientific knowledge, thus contributing to the training of undergraduate students in nutrition.

Keywords: Academic leagues; Functional nutrition; Sports nutrition; Teaching.

\section{Resumen}

La Liga Académica de Nutrición Funcional y Deportiva (LANFE) fue fundada por académicos con el propósito de mejorar los conocimientos teóricos y prácticos sobre nutrición funcional y deportiva. El objetivo de este estudio fue reportar la experiencia de crear e implementar un proyecto de extensión de la 1a liga académica (LA) en un curso de nutrición en una universidad pública del Estado de Rio de Janeiro, para discutir las actividades realizadas y evaluar su impacto. Se trata de un estudio cualitativo-cuantitativo, descriptivo, del tipo informe de experiencia de creación y primer año de actividades ligueras. En la evaluación de los cuestionarios aplicados a la comunidad interna y externa de la universidad, se observó que 99,2\% de los participantes $(n=123)$ creían en la importancia de crear una LA para el Curso de Nutrición. En cuanto a las actividades realizadas por LANFE, el $100 \%$ de los participantes $(\mathrm{n}=15)$ del workshop de gastronomia funcional sobre el cacao consideró "muy bueno" el nivel de calidad del evento, así como el apoyo a las dudas e inquietudes expuestas. Además, el 85,3\% de los participantes en el 1er Simposio LANFE $(\mathrm{n}=34)$ demostraron un grado de satisfacción "muy bueno". De esta manera, la liga académica demostró ser una importante herramienta de enseñanza y aprendizaje, movilizando a estudiantes y profesionales a favor de la construcción y puesta en común del conocimiento científico, contribuyendo así a la formación de estudiantes de pregrado en nutrición.

Palabras clave: Ligas académicas; Nutrición funcional; Nutrición deportiva; Ensenãnza.

\section{Introdução}

As Ligas Acadêmicas (LA) são criadas visando suprir possíveis deficiências na grade curricular de universidades, além de proporcionar maior contato com o tema proposto pelas ligas, de forma prática. Ainda, as LA caracterizam-se como um modelo fundamentado e organizado por discentes e coordenado por docentes (Goergen, 2017).

Desta forma, as LA são consideradas como um espaço que permite o desenvolvimento do ensino, da pesquisa e da extensão, onde ocorre o elo entre os estudantes, os profissionais e a comunidade (Silva, Almeida, Capellini \& Silva, 2020). Além disso, elas são capazes de contribuir para a formação de profissionais da área de saúde através do estímulo ao trabalho em equipe, a reflexão crítica e a autonomia dos estudantes (Cavalcante, Vasconcelos, Lira, Henriques, Albuquerque, Maciel, Ribeiro, \& Gomes, 2018).

Contudo, esse fenômeno de articulação e mobilização que teve início no século XX, apresenta-se majoritariamente entre os cursos de graduação em Medicina. Tal fato pode estar atrelado aos seus primeiros indícios se darem em prol do combate à alta prevalência de agravos à saúde oriundos da tuberculose e hanseníase presentes no começo deste século. Ainda no que tange a concentração desse movimento, a segunda onda de criação e implementação de ligas se manteve no âmbito de universidades de Medicina, que tinham como objetivo debater e aprofundar conhecimentos acerca de determinados conceitos de saúde/doença (Andreoni, Rangel, Barreto, Rodrigues, Alves, \& Portela, 2019).

No que diz respeito ao curso de graduação em Nutrição, a presença das ligas acadêmicas ainda não é uma realidade das instituições e, em sua maioria, os movimentos que surgem nessa vertente apresentam característica pioneira (Vitorino, Silva, Araújo, Lessa, Pires, Santos, Ramos, \& Teixeira, 2020). Neste contexto está inserida a criação da Liga Acadêmica de Nutrição Funcional e Esportiva (LANFE), que consiste num projeto de extensão universitário idealizado por graduandos do curso de Nutrição da Universidade do Estado do Rio de Janeiro (UERJ). A LANFE visa, por meio de suas atividades, atingir os três pilares que orientam uma LA: ensino, pesquisa e extensão (Moreira, Mennin, Lacaz, \& Bellini, 2019; Silva, et al., 2020). Sem fins lucrativos e com duração ilimitada, a LANFE se estruturou em seis cargos distintos com o intuito de otimizar a 
gestão das atividades propostas, sendo eles: presidente, vice-presidente, diretor de comunicação, diretor tesoureiro, diretor de pesquisa e diretor de extensão.

Sua idealização e criação buscou atender demandas relacionadas à formação do aluno de graduação no curso de nutrição, dentre as quais destacam-se o conhecimento técnico e familiarização com novos caminhos de atuação profissional que não são contemplados no currículo obrigatório da instituição, bem como promoção de atividades práticas que colaborem para formação profissional do discente e o aproximem da comunidade. Nesse aspecto destacam-se as áreas de atuação em Nutrição Clínica Funcional e Nutrição Esportiva, campos dentro da nutrição que vem ganhando destaque nos últimos anos. Dados do Conselho Federal de Nutricionistas (CFN) sobre o perfil desses profissionais no Brasil indicam que 30,4\% atuam no campo da Nutrição Clínica, enquanto 2,5\% estão inseridos na área da Nutrição Esportiva (Conselho Federal de Nutricionistas, 2019).

A Nutrição Clínica caracteriza-se por prestar assistência dietética e promover educação nutricional a indivíduos, sadios ou enfermos, em nível hospitalar, ambulatorial, domiciliar e em consultórios de nutrição e dietética, visando à promoção, manutenção e recuperação da saúde. Inserida no âmbito da clínica, a Nutrição Funcional busca compreender de maneira científica e integrativa a relação entre os diferentes sistemas do organismo por meio da relação entre a fisiologia, fatores emocionais, cognitivos e estruturais, no ato de avaliar aspectos genotípicos e bioquímicos individuais (Demétrio, Paiva, Fróes, Freitas \& Santos, 2011).

Ainda no que diz respeito às áreas de atuação do nutricionista, destaca-se a nutrição esportiva. Com a crescente propagação da importância atrelada à prática esportiva e o aumento do número de atletas e praticantes de atividade física, a necessidade por um profissional de saúde que atenda as demandas específicas deste público torna-se inevitável. Neste aspecto, o nutricionista tem um papel essencial na vida do praticante de atividade física ou atleta, uma vez que a performance esportiva está diretamente ligada à nutrição (Mendonça, Perin, Rodrigues, \& Costa, 2019).

Além disso, a LANFE teve como propósito adicional estimular a interdisciplinaridade entre as LA existentes no campo da saúde, sendo esse um meio de ampliar o olhar sobre o indivíduo, tanto no aspecto biológico como social, e difundir o movimento de liga acadêmica entre os demais cursos de graduação, sendo essa uma carência observada tanto na estrutura interna quanto nas atividades promovidas (Nascimento, Brito, Santos, Borges, Silva, Sakai, Ferreira, Manrique, \& Duarte, 2020).

Portanto, com o intuito de expor e analisar as principais atividades realizadas pela LA e seus impactos, o presente artigo teve como objetivo relatar a experiência de criação e implantação do projeto de extensão da primeira liga acadêmica do curso de nutrição da Universidade do Estado do Rio de Janeiro, discorrer sobre as atividades realizadas pela liga e avaliar suas repercussões.

\section{Metodologia}

Trata-se de um estudo quali-quantitativo, descritivo, do tipo relato de experiência, acerca da criação e implantação do projeto de extensão LANFE. Ele foi desenvolvido por alunos do curso de graduação em Nutrição do Campus Maracanã da UERJ e, primordialmente, direcionado aos discentes das faculdades públicas e privadas de Nutrição do Estado do Rio de Janeiro.

Primeiramente foram relatadas as atividades realizadas com o público interno e externo desde a concepção e a submissão do projeto da liga (dezembro de 2018), até o final de seu primeiro ano de atividades (dezembro de 2019).

Ademais, foi feito o levantamento de dados oriundos de cinco formulários de pesquisa de opinião elaborados através da plataforma Google Forms®. O primeiro, intitulado "Questionário de avaliação sobre o interesse dos discentes da UERJ quanto às áreas temáticas da Nutrição e criação da Liga Acadêmica no campus Maracanã”, foi desenvolvido durante a criação 
do projeto e visou avaliar, sobretudo, se os estudantes de nutrição da UERJ desejavam a criação de uma liga acadêmica e se demonstravam interesse pelas áreas de nutrição funcional e a nutrição esportiva. $\mathrm{O}$ formulário foi divulgado nas redes sociais dos fundadores da liga e apresentou, além da caracterização do perfil (sexo, idade e escolaridade), duas perguntas de múltipla escolha (uma com opção de selecionar apenas uma resposta e uma com seleção de até duas respostas).

Os demais formulários foram relativos a dois eventos realizados pela LANFE, um workshop e um simpósio, e direcionados, via e-mail, aos participantes inscritos e com presença confirmada. Um deles, intitulado “Workshop de Gastronomia Funcional sobre Cacau: da teoria à prática", foi enviado antes da realização do evento e estruturado em duas seções: a primeira, com treze perguntas de múltipla escolha, teve o objetivo de avaliar os conhecimentos prévios a respeito do tema que seria abordado (resultados não apresentados), enquanto a segunda, com onze perguntas de múltipla escolha, visou obter as expectativas em relação ao evento, além das impressões dos participantes acerca da liga acadêmica e de sua contribuição para a formação acadêmica dos alunos.

Após a realização do evento, os participantes responderam a um questionário igual ao anterior, que, nesse segundo momento foi intitulado "FEEDBACK Workshop de Gastronomia Funcional sobre Cacau: da teoria à prática", com o objetivo de avaliar os conhecimentos adquiridos no workshop, através da comparação das respostas obtidas pré e pós evento.

Para avaliar a satisfação em relação ao workshop e ao simpósio foi elaborado um modelo de questionário a ser enviado aos participantes após a ocorrência de cada evento, recebendo somente uma nomenclatura diferenciada nesses dois momentos para permitir a identificação do evento ao qual se referia: "Questionário de satisfação Workshop de Gastronomia Funcional sobre Cacau: da teoria à prática" e "Questionário de satisfação I Simpósio da LANFE". Estes foram estruturados com seis perguntas de múltipla escolha (com escala Likert unipolar - avalia a presença ou ausência de uma qualidade) sobre a qualidade, grau de satisfação e expectativas em relação ao evento em questão.

Os dados foram avaliados por estatística descritiva básica no programa Excel $365 \circledast$, onde foi avaliada a taxa de retorno (TR - \%) dos formulários referentes a eventos realizados pela liga, ou através de gráficos gerados pelo Google forms®. O cálculo da TR é feito através da divisão do número absoluto de respostas obtidas em cada formulário pelo número absoluto de participantes do evento abordado por ele. Assim, a TR do formulário "Workshop de Gastronomia Funcional sobre Cacau: da teoria à prática" foi de 100\% (15 respostas/ 15 participantes), enquanto a dos formulários "FEEDBACK Workshop de Gastronomia Funcional sobre Cacau: da teoria à prática" e "Questionário de satisfação Workshop de Gastronomia Funcional sobre Cacau: da teoria à prática" foram de 87\% (13 respostas/ 15 participantes). Já a TR do formulário "Questionário de satisfação I Simpósio da LANFE” foi de 48\% (34 respostas / 71 participantes).

\section{Resultados e Discussão}

\subsection{Criação e implantação do projeto de extensão intitulado: Liga Acadêmica de Nutrição Funcional e Esportiva (LANFE)}

Inicialmente, os seis acadêmicos de nutrição elegeram uma professora para orientá-los no processo de criação e implantação do Projeto de Extensão. A equipe de discentes elaborou um questionário de avaliação do interesse dos discentes na criação de uma liga acadêmica e sobre as áreas da nutrição que desejariam que esta contemplasse, visto a grande variabilidade de conhecimentos que podem ser abordados a partir de uma liga (Goergen, 2017). As características gerais dos indivíduos que responderam ao questionário, bem como as áreas da nutrição de interesse estão sintetizadas na Tabela 1. 
Tabela 1. Características gerais dos indivíduos que responderam ao questionário de avaliação sobre o interesse dos discentes da UERJ quanto às áreas temáticas da Nutrição e criação da Liga Acadêmica no campus Maracanã.

\begin{tabular}{|c|c|c|c|}
\hline \multicolumn{2}{|c|}{ Variáveis } & \multicolumn{2}{|c|}{ Participantes $(\mathrm{n}=123)$} \\
\hline \multirow{3}{*}{ Sexo } & & n & $\%$ \\
\hline & Feminino & 108 & 87,8 \\
\hline & Masculino & 15 & 12,2 \\
\hline \multirow{2}{*}{ Idade } & & Mediana & p25 - p75 \\
\hline & & 22 anos & $20-23$ \\
\hline \multirow{10}{*}{ Escolaridade } & & $\mathbf{n}$ & $\%$ \\
\hline & Discentes (graduação) & 107 & 87,0 \\
\hline & UERJ & 106 & 86,2 \\
\hline & Outras faculdades & 1 & 0,8 \\
\hline & Discentes (pós-graduação) & 2 & 1,6 \\
\hline & Docentes & 7 & 5,7 \\
\hline & UERJ & 6 & 4,9 \\
\hline & Outras faculdades & 1 & 0,8 \\
\hline & Profissionais da saúde & 4 & 3,2 \\
\hline & Outros & 3 & 2,4 \\
\hline \multirow{3}{*}{$\begin{array}{c}\text { Interesse na criação de uma } \\
\text { liga }\end{array}$} & & $\mathbf{n}$ & $\%$ \\
\hline & Sim & 122 & 99,2 \\
\hline & Não & 1 & 0,8 \\
\hline \multirow{7}{*}{ Áreas da nutrição de interesse } & & $\mathbf{n}$ & $\%$ \\
\hline & Nutrição Clínica & 71 & 57,7 \\
\hline & Nutrição Esportiva & 59 & 48,0 \\
\hline & Nutrição Funcional & 56 & 45,5 \\
\hline & Saúde Coletiva & 19 & 15,4 \\
\hline & Alimentação Coletiva & 17 & 13,8 \\
\hline & Outros & 11 & 8,9 \\
\hline
\end{tabular}

Fonte: Autores (2021).

Observou-se grande interesse pelas áreas que os idealizadores da liga pretendiam abordar, onde 48\% dos participantes sinalizaram interesse pela Nutrição Esportiva, seguida da Nutrição Funcional, que atingiu um total de 45,5\%, demonstrando uma necessidade dos discentes para construção de uma liga acadêmica que abordasse os respectivos temas. Entre outras sugestões estavam temas relacionados à Agroecologia, Nutrição Infantil, Nutrição Comportamental e Ciência e Tecnologia de Alimentos.

Além disso, 99,2\% dos participantes que responderam ao questionário desejavam a criação de uma LA para o Curso de Nutrição, o que corrobora com o desejo dos alunos envolvidos na gestão de criar a primeira liga acadêmica do curso de nutrição da UERJ. Esse formato de projeto de extensão antes era visto predominantemente nos cursos de medicina, essencialmente de universidades públicas (Silva, et al., 2020).

Assim definiram-se as áreas do saber em foco do projeto, a Nutrição Funcional e a Esportiva, para realização das atividades da liga acadêmica, dando origem à LANFE. O projeto desenvolveu saberes nestas áreas com base no tripé universitário: o ensino, a pesquisa e a extensão, de acordo com as Diretrizes Curriculares Nacionais, para alcançar o ensino crítico-reflexivo dos discentes envolvidos com o projeto de extensão, por meio das atividades realizadas pelos mesmos, aproximando os estudantes e a população (Araujo, Lopes, Dias, Neto, Farias, \& Cavalcante, 2019).

Para isso, foram realizadas reuniões entre os alunos e a professora coordenadora para discutir como seria a implantação do projeto e elaborar em conjunto o Estatuto para o estabelecimento de normas para o funcionamento e as atividades da liga. Nele estão descritos os fundadores, diretores, efetivos, supervisores, colaboradores, a constituição da Assembleia, os cargos de diretores com seus deveres e funções, além do funcionamento do projeto. Foram definidos os 
seguintes cargos para a equipe: Diretor Presidente, Diretor Vice-Presidente, Diretor Tesoureiro, Diretor de Pesquisa, Diretor de Comunicação e Diretor de Extensão. Essa organização estimula a independência e a liberdade dos estudantes inseridos na gestão, desenvolvendo a responsabilidade, liderança, autonomia e habilidades de gestão (Yang, Braga, Hipólito, Vieira, Pessanha, Abrantes, \& Corrêa, 2019).

Além do Estatuto, foi desenvolvido o logotipo do projeto para promoção de uma identidade visual promotora de identificação da liga (Figura 1). O símbolo reflete o estado de bem estar proporcionado pelos pilares da LANFE: alimentação e exercício, sendo composto pela imagem de um indivíduo em movimento formada por pares de bases nitrogenadas do DNA, que por meio de suas cores, remetem à importância da diversidade alimentar na prática de exercícios físicos.

Figura 1. Logotipo da Liga Acadêmica de Nutrição Funcional e Esportiva.

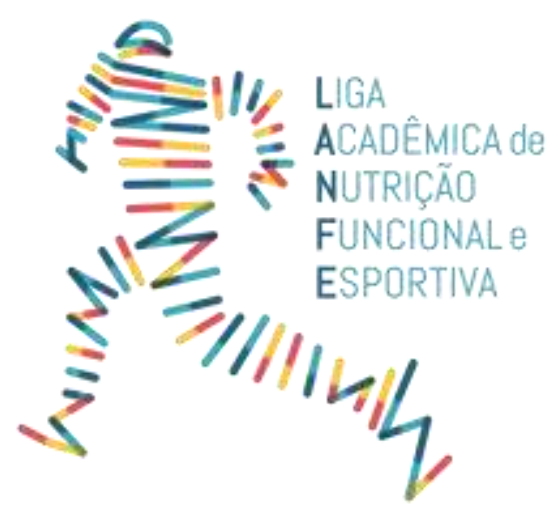

Fonte: Autores (2021).

A inserção prévia do aluno na prática profissional e em assuntos de recente conhecimento científico, propicia um melhor entendimento dos conteúdos teóricos das variadas disciplinas da graduação bem como a integralidade das mesmas, o que demonstra uma melhor adaptação ao método de ensino das ligas acadêmicas quando comparado ao ensino tradicional da graduação (Moreira, et al., 2019; Yang, et al., 2019).

A LANFE teve como propósito o desenvolvimento, a promoção e a difusão de conhecimentos acerca da área Nutrição Funcional e Esportiva, contribuindo para a formação acadêmica e contato profissional dos alunos a ela vinculados e buscou a integração da universidade para com a comunidade externa. Isso envolveu trabalhar os eixos nutrição, esporte e metabolismo de forma transversal, tendo como base os conhecimentos científicos das respectivas áreas, além de fomentar debates e discussões que visassem aprimorar o conhecimento científico dos ligantes e participantes da liga, auxiliando no âmbito social através de projetos de extensão que veiculassem o ensino e a promoção da saúde através da nutrição.

Após determinar as áreas de foco da Liga Acadêmica, foi escrito o projeto de extensão intitulado "Liga Acadêmica de Nutrição Funcional e Esportiva" que foi submetido à Sub-reitoria de Extensão e Cultura (SR3), atualmente, Pró-reitoria de Extensão e Cultura (PR3). Depois de aprovado, foram feitos os canais de comunicação virtual do projeto no Instagram ${ }^{\circledR}$ e no Facebook ${ }^{(F i g u r a ~} 2$ e 3). 
Figura 2. Canal de comunicação virtual do projeto no Instagram®.

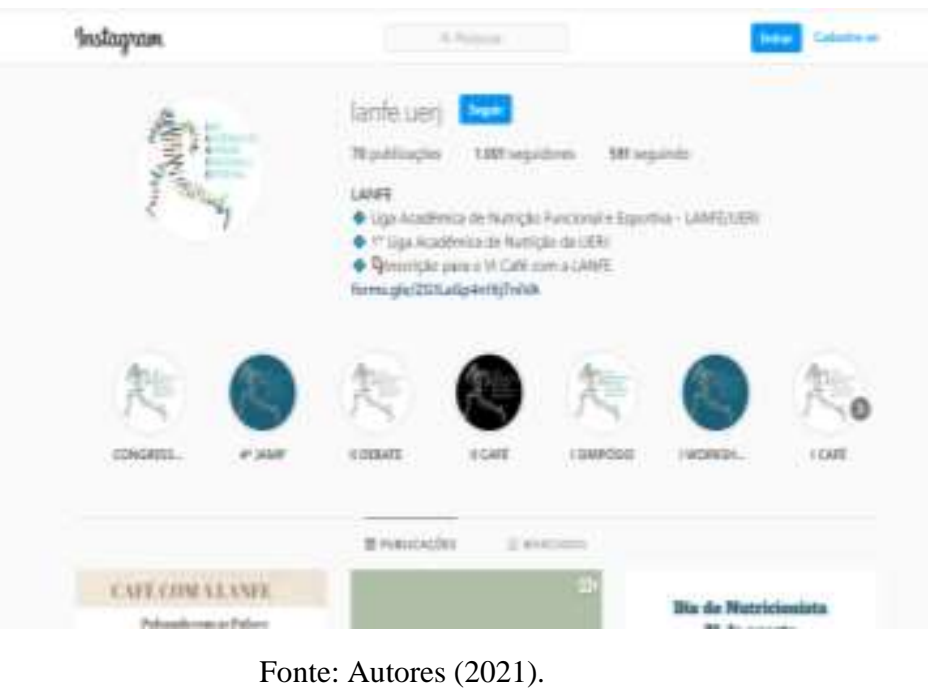

Figura 3. Canal de comunicação virtual do projeto no Facebook®.

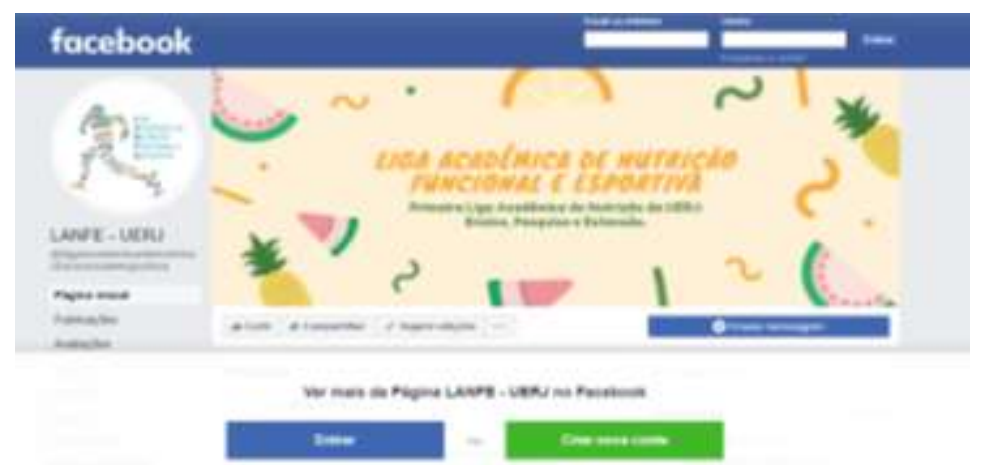

Fonte: Autores (2021).

Para iniciar as ações promovidas pela LANFE foi realizado o processo seletivo de membros efetivos (ligantes) para se tornarem parte da gestão do projeto. Os alunos foram selecionados a partir de entrevistas conduzidas pelos discentes responsáveis pela criação e implantação do projeto. Inicialmente, a diretoria da Liga selecionou cinco alunos para participarem do projeto como membros efetivos, com direitos e deveres de acordo com o regimento do Estatuto, tornando-se parte da gestão a fim de organizar, planejar e participar das atividades que seriam realizadas pela Liga Acadêmica. Além disso, no segundo semestre de atividade, foram abertas novas inscrições para dar oportunidade a um maior número de alunos, onde novos membros, da própria universidade e de outras, se tornaram ligantes.

\subsection{Atividades realizadas pela Liga Acadêmica de Nutrição Funcional e Esportiva (LANFE)}

A realização das atividades desenvolvidas pela LANFE no primeiro ano (Figura 4) iniciou com foco no eixo do Ensino, através de aulas expositivas, discussões de artigos científicos, organização de eventos com ênfase nas áreas de interesse do projeto e posteriormente, buscou-se atingir os pilares de pesquisa e extensão. Sabe-se que a contribuição das LA busca contemplar o tripé da universidade e desta forma as atividades oferecidas vêm se destacando no ambiente universitário em virtude do seu potencial de contribuição no processo de ensino-aprendizagem (Tedeschi, Rigolon, Mendes, Fischmann, Klein, \& Baltar, 2018). 
Figura 4. Atividades realizadas pela LANFE em seu primeiro ano de execução.

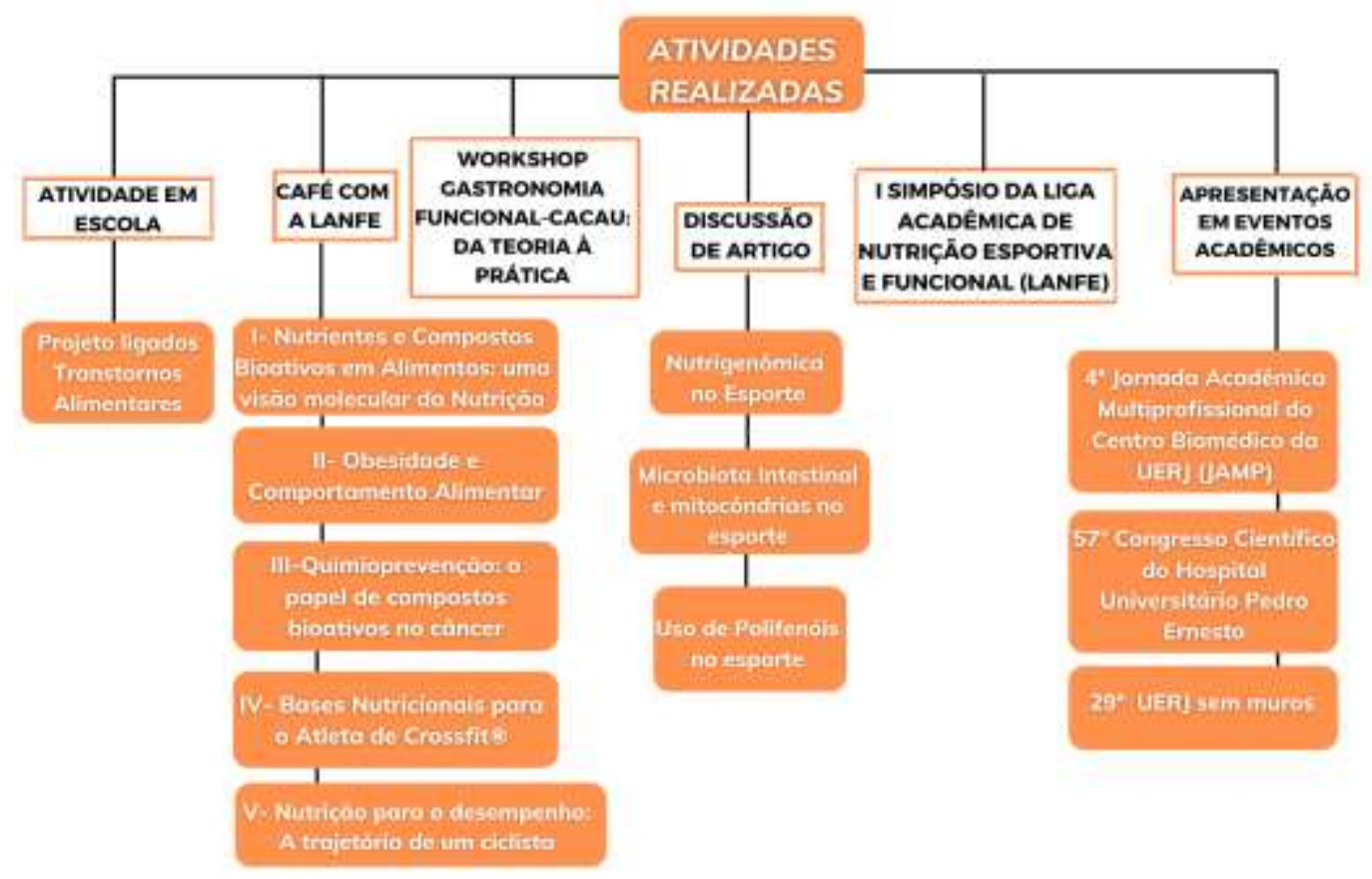

Fonte: Autores (2021).

Inicialmente, as atividades eram compostas por um debate científico mensal realizado com a gestão da LA e seus membros efetivos, sobre temas referentes à Nutrição Funcional e/ou Esportiva. O primeiro debate científico foi sobre Nutrigenômica no Esporte, oportunizando uma série de discussões sobre a ciência da nutrição e a aplicação na prática clínica para os(as) nutricionistas. Neste encontro foram levantados pontos a respeito da Nutrição de Precisão e sua importância no desempenho de atletas de alto rendimento.

Em seguida, realizou-se o segundo debate científico, a discussão contemplou a relação entre a microbiota intestinal e mitocôndrias no esporte, que buscou abordar a relevância do ambiente intestinal sobre uma das principais adaptações quando o assunto é otimização do metabolismo energético. Ainda, aconteceu outra reunião científica sobre novas evidências do uso de polifenóis no esporte.

Além dos encontros em que se debatiam evidências científicas atuais sobre Nutrição Funcional e Esportiva, a LA também realizou palestras para a comunidade interna e externa da universidade e organizou eventos como Workshop e Simpósio abertos ao público. É importante ressaltar que essas atividades de Ensino não realizavam distinções de conhecimento prévio dos assuntos que seriam abordados nas aulas expositivas, o que não limitava a participação de qualquer interessado nos temas das atividades.

As palestras abertas ao público geral foram organizadas por meio de convites aos palestrantes, que podiam ser tanto do corpo docente da própria universidade, quanto professores e nutricionistas externos, desde que esses fossem tidos como referências na área de interesse. Os temas eram definidos em colaboração com os palestrantes que aceitaram o convite para atender os interesses dos participantes do projeto, bem como os ouvintes que desejavam aprender sobre os assuntos com foco na Nutrição Funcional e Esportiva.

Essas palestras eram intituladas "Café com a LANFE". A primeira palestra aberta ao público geral aconteceu em um auditório do Instituto de Nutrição (INU) da UERJ, com o tema "Nutrientes e Compostos Bioativos em Alimentos: uma visão molecular da Nutrição", conduzida por um professor convidado do INU. A segunda palestra para a comunidade universitária 
interna e externa foi sobre o tema "Obesidade e Comportamento Alimentar", ministrada por uma professora convidada do INU/UERJ.

Ainda foi realizado um Café com a LANFE com um tema relacionado ao "Outubro Rosa", uma campanha de conscientização que tem como foco principal a luta contra o câncer de mama, estímulo à participação da população no combate a essa doença, proporcionar maior acesso aos serviços de diagnóstico e de tratamento contribuindo assim para a redução da mortalidade (Pontes, Quitete, Reis, Silva, Silva, Silveira, Cerqueira, \& Castro, 2020). A palestra buscou abranger a Nutrição Clínica com a abordagem da Nutrição Funcional, com a apresentação intitulada "Quimioprevenção: o papel de compostos bioativos no câncer", realizada por uma aluna de doutorado do Programa de Pós-Graduação em Alimentação, Nutrição e Saúde da UERJ.

Com foco na Nutrição aplicada ao Esporte, a LANFE promoveu palestras com interesse na área esportiva, como "Bases Nutricionais para o Atleta de Crossfit ${ }^{\circledR}$ " e outra com o tema "Nutrição para o desempenho: a trajetória de uma ciclista", ambas ministradas por nutricionistas com experiência nos seus respectivos temas.

Em relação ao Workshop, o tema e os palestrantes foram debatidos e escolhidos em reunião com a professora coordenadora do projeto, os alunos da gestão e os membros efetivos. O tema escolhido foi Gastronomia Funcional e o evento foi intitulado "Workshop de Gastronomia Funcional - Cacau: da teoria à prática”, com 15 vagas para atender o número limite de participantes no laboratório na parte prática.

O Workshop de Gastronomia Funcional foi ministrado pela coordenadora da liga acadêmica. A parte teórica contou com uma aula expositiva com uma vasta literatura científica acerca do tema, enquanto a atividade prática foi realizada no Laboratório de Técnica Dietética do INU da UERJ. Os participantes foram divididos em quatro bancadas e em cada uma elaboraram duas receitas com cacau, propiciando aplicar o conhecimento obtido anteriormente na aula expositiva. Além disso, todos os participantes realizaram uma análise sensorial de chocolates de diferentes percentuais de cacau para conhecerem a diversidade dos teores dessa matéria-prima.

Outro evento organizado pela LANFE foi o "I Simpósio da Liga Acadêmica de Nutrição Funcional e Esportiva (LANFE)" cujo objetivo foi reunir nutricionistas reconhecidos nas áreas de Nutrição Funcional e Esportiva para ministrar as palestras com imersão no tema por alunos e profissionais interessados, tanto da comunidade interna como externa, como uma forma de reflexão e construção de conhecimento que possa atender às questões de relevância social (Cavalcante, et al., 2018).

O I Simpósio da LANFE contou com a participação de nutricionistas reconhecidas nas áreas de Nutrição Funcional e Esportiva que ministraram palestras sobre diversos assuntos de destaque. Por ter sido um evento aberto ao público geral, alcançou proporções significativas, despertando o interesse da comunidade interna e externa, formada por alunos de graduação e profissionais como Nutricionistas e Educadores Físicos, graduados em universidades públicas e privadas do estado do Rio de Janeiro.

Além disso, a equipe da LANFE apresentou oralmente e publicou em anais científicos as atividades realizadas no seu primeiro semestre de criação e implantação do projeto. O trabalho "O pioneirismo da Liga Acadêmica de Nutrição Funcional e

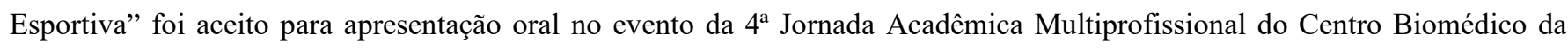
UERJ (JAMP), o qual buscou abrir o diálogo e promover integração entre os diversos cursos da área da saúde da universidade, e teve como foco o desenvolvimento das LA como um instrumento que permite a multidisciplinaridade dentro do campo das ciências biomédicas.

Ademais, houve participação da LANFE no $57^{\circ}$ Congresso Científico do Hospital Universitário Pedro Ernesto, realizado no espaço da Tarde das Ligas, com apresentação do trabalho intitulado "A importância da Liga Acadêmica de Nutrição Funcional e Esportiva (LANFE) na formação de futuros nutricionistas do estado do Rio de Janeiro". O projeto de extensão também foi apresentado em formato de banner na $29^{\circ}$ UERJ Sem Muros, um evento que visa a exposição das 
atividades de extensão que acontecem na universidade. Sendo assim, os alunos de graduação envolvidos na LANFE foram estimulados a elaborar resumos para eventos científicos, bem como apresentar os resultados obtidos no projeto em eventos, alcançando a divulgação científica das atividades realizadas, assim como a participação complementar na pesquisa, mesmo sem espaço de iniciação científica.

Como atividade de extensão voltada para a comunidade externa da universidade, os ligantes da LANFE participaram de uma atividade em uma escola pública do estado do Rio de Janeiro, no bairro Tijuca. A atividade foi realizada a partir do projeto "ligados" que reuniu algumas ligas acadêmicas da UERJ com o objetivo de levar os conhecimentos destas para além dos "muros" da universidade. O tema trabalhado entre os membros efetivos da LANFE e os alunos da escola foi "Transtornos Alimentares". Sabe-se que as atividades de educação em saúde e atividades assistenciais são estratégias utilizadas pelas LA para promover extensão universitária (Silva, et al., 2020).

\subsection{Avaliação do impacto das atividades realizadas pela Liga Acadêmica de Nutrição Funcional}

Dentre as atividades realizadas pela LANFE, para o evento "Workshop de Gastronomia Funcional sobre Cacau: da teoria à prática", avaliado a partir das respostas dos participantes $(\mathrm{n}=15)$ a um questionário enviado antes do workshop ocorrer, observou-se que $100 \%$ dos alunos afirmaram que a LANFE era um projeto que contribuía para a Universidade e para formação acadêmica, capaz de congregar acadêmicos da área da saúde interessados no aprendizado e no desenvolvimento técnico científico na área de Nutrição, propagando, assim, conhecimentos científicos acerca de questões atuais da nutrição esportiva e funcional. Quanto às perguntas sobre o impacto que o evento teria para os alunos, todos os participantes $(\mathrm{n}=15)$ afirmaram acreditar que o workshop iria contribuir para o seu aprendizado, sendo para sua formação e/ou atualização, visando ampliar o conhecimento da nutrição clínica funcional.

Vinte cinco porcento $(25 \%)$ dos participantes da pesquisa $(n=4)$ acharam que a liga e o workshop talvez viessem com propósito de estimular e incentivar a adoção de bons hábitos alimentares e a inclusão de alimentos funcionais na comunidade universitária e público em geral. Enquanto $12,5 \%(\mathrm{n}=2)$ acreditavam que talvez o workshop proporcionaria aplicação de conceitos cientificamente comprovados a fim de proporcionar bem estar e qualidade de vida à população e 6,3\% $(\mathrm{n}=1)$ dos participantes responderam que talvez a liga e o workshop visassem atender aos pilares que estruturam a Extensão Universitária, com temas atuais e promissores, contribuindo para obtenção de futuros estudos na promoção da saúde na população brasileira por meio da Nutrição.

Após a realização do workshop, os participantes responderam a um questionário que continha as mesmas perguntas que o anterior, a fim de permitir a comparação pré e pós evento. Assim, foi observado que $100 \%$ dos participantes tomaram conhecimento da história do cacau, matéria-prima destaque do workshop, bem como dos diferentes tipos e variedades disponíveis. Os resultados foram satisfatórios ao analisar a autoavaliação dos participantes em relação aos itens descritos anteriormente. Dessa forma, 92,3\% alegaram conhecer a terminologia "Xocóatl", o processo tecnológico de obtenção do chocolate, assim como os diferentes produtos que podem ser obtidos a partir dessa matéria-prima e os compostos bioativos característicos. Além disso, 84,6\% julgavam-se capazes de distinguir as diferenças entre os principais tipos de cacau, o teor presente nas opções de chocolate disponíveis no mercado e as legislações norteadoras dos processos produtivos do chocolate e produtos oriundos do cacau. Por fim, buscando compreender o impacto do workshop sobre atividades práticas, 92,3\% dos participantes sinalizaram a participação em preparações culinárias que tinham como base o chocolate.

Em último momento, foi enviado um questionário de satisfação que teve como intuito avaliar o nível de satisfação dos inscritos, assim como identificar em concordância com as respostas do primeiro documento enviado se, de fato, o evento supriu as expectativas e demandas sinalizadas previamente. Com isso, observou-se que $100 \%$ dos participantes demonstraram satisfação e nível de qualidade "muito bom", sendo a escala de muito ruim até muito bom, ao evento realizado, assim como 
suporte às dúvidas e preocupações expostas. Desses, 76,9\% consideraram a experiência muito melhor do que a esperada, enquanto $61,5 \%$ consideraram extremamente claras as informações transmitidas e a adequação do cronograma previamente divulgado com as expectativas geradas. Dessa forma, 92,3\% e 76,9\% indicaram numa escala de 0 a 10 , 100\% de possibilidade em indicar as atividades, ações e eventos realizados pela liga a amigos ou conhecidos e a intenção de participar novamente das mesmas, respectivamente.

Além do workshop, também foi realizado o I Simpósio da Liga Acadêmica de Nutrição Funcional e Esportiva, cuja aceitação geral foi avaliada por meio de um questionário de satisfação intitulado "Questionário de satisfação I Simpósio da LANFE". Através deste, observou-se que a maioria (85,3\%) dos participantes demonstraram um grau de satisfação "muito bom" em uma escala que variava de muito bom a muito ruim. Quanto ao suporte a dúvidas e preocupações expostas, dentro de uma escala que variava do muito bem ao muito mal, 91,2\% declarou que o simpósio foi capaz de saná-las muito bem. Ainda, a maioria $(64,7 \%)$ dos participantes considerou a experiência com o evento muito melhor do que a esperada, seguido por moderadamente melhor (26,5\%). Quanto à clareza das informações fornecidas no simpósio, metade do público considerou extremamente claras, enquanto a outra metade julgou muito $(44,1 \%)$ ou moderadamente $(5,9 \%)$ claras. Além disso, em uma escala de 0 a 10 (onde o 0 representa nenhuma probabilidade e o 10 indica 100\% de probabilidade) sobre a possibilidade de indicar as atividades, ações e eventos realizados pela liga a amigos ou conhecidos e a intenção de participar novamente das mesmas, 76,5\% selecionaram a opção "10".

\section{Considerações Finais}

Tendo em vista todos dados dispostos no presente estudo, fica claro que a LANFE pode ser considerada um importante instrumento na formação dos estudantes de Graduação em Nutrição, uma vez que, desde a sua criação foi pensada para contribuir com a formação e atualização dos participantes e a partir da sua implantação, a realização das atividades do projeto e avaliação do impacto das mesmas, pode-se observar que a liga agregou conhecimento em nutrição funcional e esportiva, sendo capaz de ampliar o conhecimento nestas áreas e corroborando a sua importância para a formação de futuros nutricionistas.

\section{Referências}

Alves, E., Rossi, C. E., \& Vasconcelos, F. A. G. (2003). Nutricionistas egressos da Universidade Federal de Santa Catarina: áreas de atuação, distribuição geográfica, índices de pós-graduação e de filiação aos órgãos de classe. Revista de Nutrição, 16 (3), 295-304. https://doi.org/10.1590/S141552732003000300007

Andreoni, S., Rangel, D. C., Barreto, G. C. B. G. de S., Rodrigues, R. H. I., Alves, H. M. T., \& Portela, L. A. (2019). O perfil das ligas acadêmicas de angiologia e cirurgia vascular e sua eficácia no ensino da especialidade. Jornal Vascular Brasileiro, 18 (0), 1-5. 10.1590/1677-5449.006318

Araujo, C. R. C., Lopes, R. E., Dias M. S. A., Neto, F. R. G. X., Farias, Q. L. T., \& Cavalcante, A. S. P. (2019). Contribuição das ligas acadêmicas para Formação em enfermagem. Enfermagem em Foco, 10 (6). https://doi.org/10.21675/2357- 707X.2019.v10.n6.2802

Cavalcante, A. S. P., Vasconcelos, M. I. O., Lira, G. V., Henriques, R. L. M., Albuquerque, I. N. M., Maciel, G. P., Ribeiro, M. A., \& Gomes, D. F. (2018). As ligas acadêmicas na área da saúde: lacunas do conhecimento na produção científica brasileira. Revista Brasileira de Educação Médica, 42 (1), $197-204$. https://doi.org/10.1590/1981-52712018v42n1rb20170081

Conselho Federal de Nutricionistas. (2019). Perfil do Nutricionista Brasileiro. http://pesquisa.cfn.org.br/

Dantas, A. C. de O., Santos, M. A., Gois, M. B. T. C. (2017). Importância da liga acadêmica para a formação profissional: aprendendo a trabalhar em equipe. International Nursing Congress, 1 (1), 1-3. https://eventos.set.edu.br/cie/article/view/6154/2429

Demétrio, F., Paiva, J. B. de, Fróes, A. A. G., Freitas, M. do C. S. de, \& Santos, L. A. S. (2011). A nutrição clínica ampliada e a humanização da relação nutricionista-paciente: contribuições para reflexão. Revista de Nutrição, 24 (5),743-763. https://doi.org/10.1590/S1415-52732011000500008

Goergen, D. I. (2017). Ligas acadêmicas: uma revisão de várias experiências. Arquivos Catarinenses de Medicina, 46 (3), 183-193. http://acm.org.br/acm/seer/index.php/arquivos/article/view/68/187 
Research, Society and Development, v. 10, n. 2, e19910212426, 2021

(CC BY 4.0) | ISSN 2525-3409 | DOI: http://dx.doi.org/10.33448/rsd-v10i2.12426

Mendonça, T. R. dos S., Perin, J. R., Rodrigues, A. P. do S., \& Costa, T. N. F. da. (2019). Nutrição esportiva como componente curricular nos cursos de nutrição e educação física da região norte do Brasil. RBNE - Revista Brasileira De Nutrição Esportiva, 13 (81), 611-616. http://www.rbne.com.br/index.php/rbne/article/view/1389

Miranda, L. E. C., Miranda, A. C. G., Lima, D. L. de, \& Arraes, A. K. A. (2020). Lessons learned from the student's surgery academic league: Is it worth it?. Revista Brasileira De Educação Médica, 44 (1). https://doi.org/10.1590/1981-5271v44.1-20190197

Moreira, L. M., Mennin, R. H. P., Lacaz, F. A. de C., \& Bellini, V. C. (2019). Ligas acadêmicas e formação médica: estudo exploratório numa tradicional escola de medicina. Revista Brasileira de Educação Médica, 43 (1), 115-125. https://doi.org/10.1590/1981- 52712015v43n1rb20170141

Nascimento, M. A., Brito, A. J., Santos, I. A., Borges, M. S., Silva, D. S., Sakai, J. D. V., Ferreira, I. C. N., Manrique, E. J. C., \& Duarte, L. S. (2020). Abordagem multiprofissional para o paciente acometido por pênfigo vulgar. Research, Society and Development, 9 (8), 4-6. http://dx.doi.org/10.33448/rsdv9i8.6596

Pontes, B. F., Quitete, J. B., Reis, R. F. dos, Silva, B. M. S. da, Silva, M. T. da, Silveira, S. S. D. da, Cerqueira, M. D. R. A. de \& Castro, R. de C. (2020). Outubro rosa: uma ação de cuidado no cenário das políticas públicas. Brazilian Journal of Development, 6 (6), $34504-34518$. https://doi.org/10.34117/bjdv6n6-117

Silva, D. A. da, Almeida, C. L. de, Capellini, V. K., \& Silva, R. G. da. (2020). Educação em enfermagem: criação de uma liga acadêmica para o ensino de urgência e emergência. Research, Society and Development, 9 (3), 1-11. http://dx.doi.org/10.33448/rsd-v9i3.2656

Silva, S. S. F. da, Cavalcante, C. B. T. L., Anizio, M. de S., Nunes, B. L. R., Pinto, A. C. S., \& Paula, D. G. de. (2020). Profile and production of academic health science leagues in Brazil: an integrative review. Research, Society and Development, 9 (9), 743997775. https://doi.org/10.33448/rsd-v9i9.7775

Tedeschi, L. T., Rigolon, L. P. J., Mendes, F. D. O., Fischmann, M., Klein, I. D. A., \& Baltar, V. T. (2018). A experiência de uma liga acadêmica: impacto positivo no conhecimento sobre trauma e emergência. Revista do Colégio Brasileiro de Cirurgiões, 45 (1). http://doi.org/10.1590/0100-6991e-20181482

Vitorino, R. S., Silva, J. H. da, Araújo, G. F. A. dos, Lessa, J. R., Pires, M. B. F., Santos, G. B. P. dos, Ramos, V. F., \& Teixeira, M. T. (2020). Experiências de uma Liga Acadêmica de Nutrição e Alimentação Materno Infantil: Um Relato de Caso. SEMEAR: Revista de Alimentação, Nutrição e Saúde. 2 (1), 1 - 7. Recuperado de http://seer.unirio.br/index.php/ralnuts/article/view/10836/9315

Yang, G. Y. H., Braga, A. C. B., Hipólito, N. da C., Vieira, K. S. T., Pessanha, C. G., Abrantes, F. G., \& Corrêa, C. L. (2019). Liga de Anatomia Aplicada (LAA): as Múltiplas Perspectivas sobre Participar de uma Liga Acadêmica. Revista Brasileira de Educação Médica, 43 (1), $80-86$. https://doi.org/10.1590/1981-52712015v43n1rb20170146 